\title{
LOW FREQUENCY NOISE GENERATED BY AN OPEN WINDOW IN A CAR AND ITS EFFECT ON HUMAN COMFORT
}

\author{
Stanislav Žiaran, Assoc Prof. Ing. PhD \\ Department of Applied Mechanics and Mechatronics, Faculty of Mechanical Engineering STU, \\ Námestie slobody 17, 81231 Bratislava, Slovak Republic, Stanislav.ziaran@stuba.sk
}

Keywords: flow, car, tonal noise, effect, human

\begin{abstract}
The paper presents low frequency noise and infrasound excited by air flow due to an open window in a moving passenger car. This noise is analysed and its effects on a driver's comfort are assessed. The results from all measurements (using FFT, as well as CPB analysis) show, that an open window at high speeds behaves as a source of specific tonal low-frequency noise, which is annoying, since the sound levels are relatively high. This strong low-frequency noise is analysed from the view point of its effects on the health of the exposed subjects and some recommendations are given.
\end{abstract}

\section{INTRODUCTION}

The air circulation in a car can be influenced by a variety of possibilities. Either the built-in air-conditioning can be used, or the air can be exchanged by opening the windows. Many drivers prefer the second option, due to some reported effects of air-conditioning on health. However, opening the windows, and so exchanging the air, leads to a reduction in the acoustic comfort of the driver and passengers, especially due to the induction of low-frequency noise. This effect was observed especially on highways, or roads out of cities. In a city the effect of wind induced noise is insignificant due to low car speeds. Under certain conditions, this specific noise can have a negative impact on the health of the driver and/or passengers. It can lead to annoyance, tiredness, lower attention to the situation around the car and an overall reduction in the drivers cognitive awareness. The result of this specific noise can have a negative impact on driving safely, especially on long-distance drives. The methodology presented in the article can be applied also for other sources of very low frequency acoustical vibration, such as airconditioning systems, boiler systems, large low-frequency Diesel engines, wind turbines, etc.

\section{CONDITIONS OF MEASUREMENTS}

The noise level was measured inside of the passenger cars NISSAN TIIDA and LEXUS. During the measurements, the cars were driving on Slovak highways with minimal traffic, in order to minimize the influence of other sources of noise from passing cars. The measurements were done at various car speeds ranging from $70 \mathrm{~km} / \mathrm{h}$ to $150 \mathrm{~km} / \mathrm{h}$ on roads chosen to be as homogeneous as possible. Another variable parameter in the analysis is the amount of window opening, where three cases are to be compared: all windows closed, window partially open (approx. $5 \mathrm{~cm}$ ) and window fully open. The noise was measured at the ear level of the driver, i.e. the microphone was positioned close to the head, in order to analyse the impact of the noise on the driver while driving the car, as shown in Fig. 1 [4, 14]. 


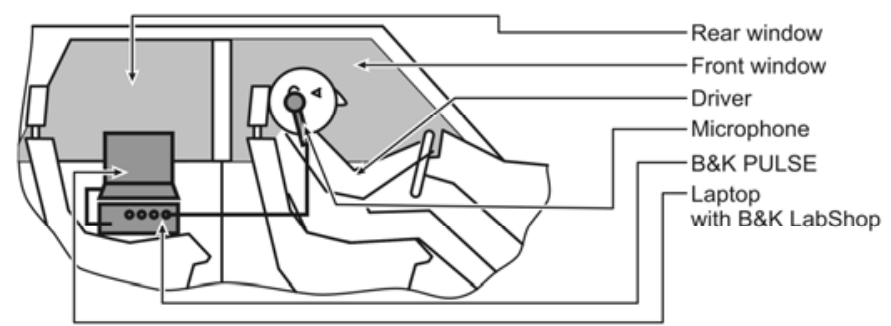

Figure 1: Schematic of the measurement set-up inside of a passenger car while driving

\section{EVALUATION OF FREQUENCY SPECTRA}

In the first set of measurements, the conditions at which the maximal level of the noise is generated were investigated. The CPB analysis was done and it was found that the dominant very low-frequency noise was generated with a fully open rear window of the car $[4,9,14]$.

The FFT measurements show that the measured data are consistent and the dispersion of peak values was a maximum of $3 \mathrm{~dB}$, as presented in Fig. 2. This difference can be caused by the speed variation of the car or variation of the air speed around the car. Similarly the frequency variation of up to $1 \mathrm{~Hz}$, at approximately the same car speed can be caused especially by the real conditions of the air stream during measurements.

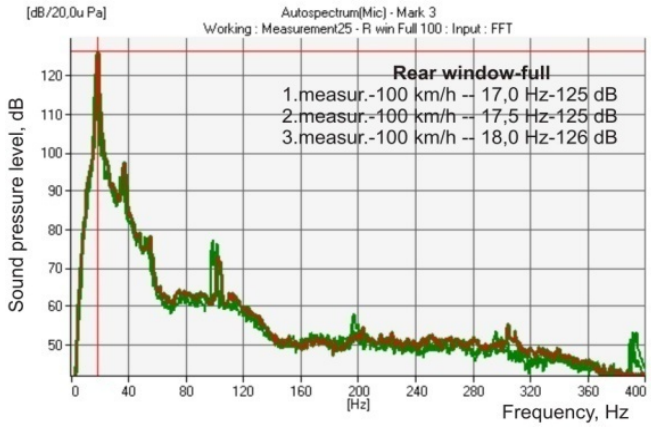

a)

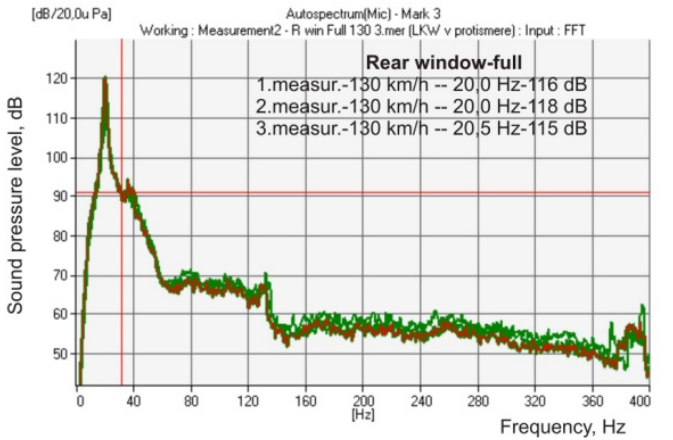

b)

Figure 2: FFT analysis of the generated noise in the car interior - three measurements with two different car speeds: a) $100 \mathrm{~km} / \mathrm{h}$; b) $130 \mathrm{~km} / \mathrm{h}$

From the FFT analysis it is obvious that due to window opening, the strong tonal very lowfrequency acoustic vibration is generated in the lower boundary of the sound perception of a human ear. The non-weighted values (so-called Z-weighting) exceeded $115 \mathrm{~dB}$, depending on car speed. These levels of sound pressure are close to the threshold of pain.

The utilization of the Z-weighting (i.e. no weighting) shows the exposition of the human being directly to this noise, regardless of the sensitivity of his/her ears. Currently there is a discussion about the evaluation of the low frequency noise of high sound pressure levels, since the A-filter, which are used most often, do not correct reflect the influences on the health and comfort of human beings [3, 5, 6, 7, 8, 12, 13]. Therefore, in analysing the measured spectra the A-, C- and Z- weights are to be presented.

Frequency analysis of the investigated low-frequency region with the application of these weightings are presented in Fig. 3, where Fig. 3a shows the results for constant speed and Fig. 3b for different speeds. The sensitivity of the human ear at low frequencies is much lower, therefore the measured results, weighted using the A- as well as C- or Z-weightings, are significantly 
different. The energy difference between the C- and A-weighting is approximately 32000 -fold, where the difference between Z- and A-weighting is up to 160000 -fold [13]. Even keeping in mind that the acoustic energy is negligible compared to other sources of energy, the presented differences in acoustical weighting should not be ignored in evaluation of the influence of the lowfrequency acoustic vibration on human beings. From the view point of human health, each type of energy has the ability to do work - either negative or positive. However, there exists a limit of positive and negative influences on the human organism, and so this limit should be set exactly or should be estimated in the most precise way.

The behaviour of the A- and C- weighting of the analysed, strong, very low-frequency acoustic vibration is presented in Fig. 4a and b. Again, there are significant differences between the A- and C- weighting when comparing the measured cases of a fully and partially open rear (on driver's side) car window (the same window used) within the frequency band of interest (10 $\mathrm{Hz}-70$ $\mathrm{Hz})$.

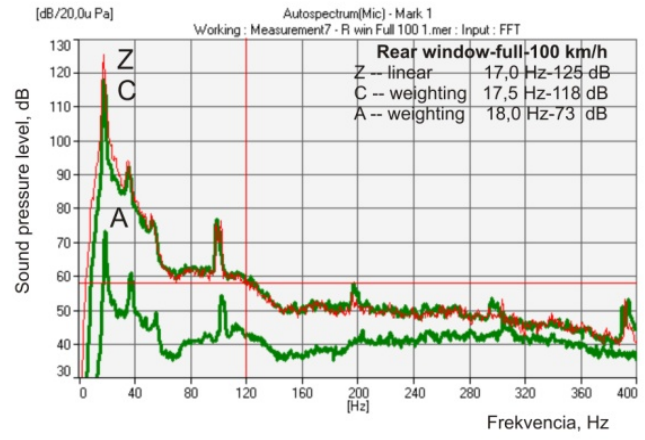

a)

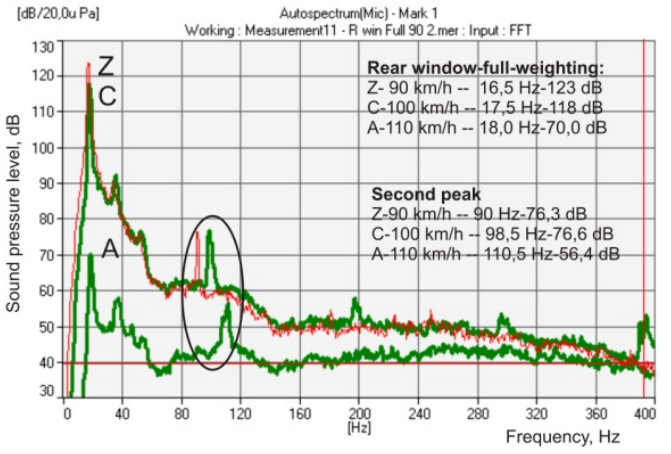

b)

Figure 3: Comparison of energies using Z-, C- and A- weighting of the same acoustic signal with different car speeds: a) $100 \mathrm{~km} / \mathrm{h}$; b) different speeds.

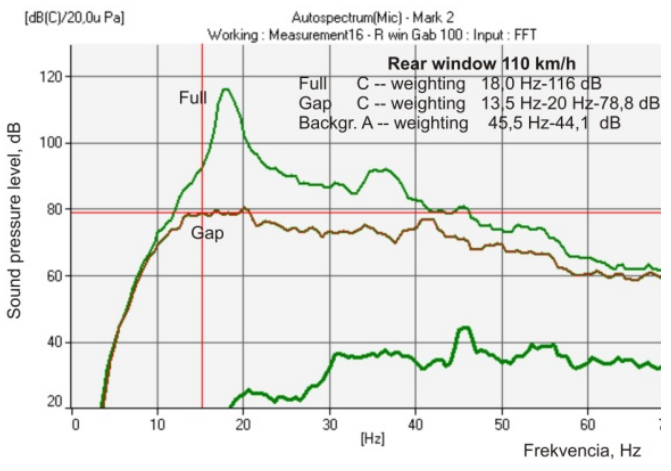

a)

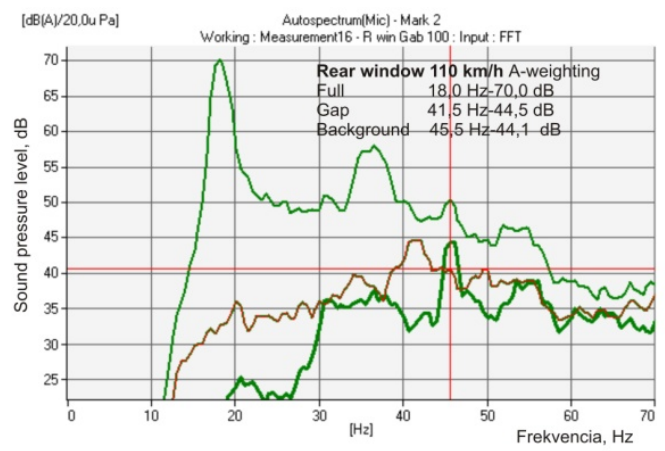

b)

Figure 4: Comparison of energies using A- and C- weighting of the same acoustic signal with the same car speed ( at $110 \mathrm{~km} / \mathrm{h}$ )

The reduction in acoustic level when applying $\mathrm{C}$ - and A-weighting, with the same maximal window opening is up to $46 \mathrm{~dB}$, whereas with a partially open rear window, the maximal noise levels are shifted to higher frequency for the A- weighting used. Even though the lowfrequency content of the acoustic energy is significantly higher than the background noise (i.e. all windows closed), as shown in Fig.4b. It is important to notice that the subjective perception of the 
driver and operator on the noise was significantly higher than the measured A-weighted sound pressure, and this perception was reflected more so in the acoustic C- weighting.

A Similar behaviour of the frequency spectra was also analysed at higher car speeds, where the difference between $\mathrm{C}$ - and A- weighting was just $2 \mathrm{~dB}$ lower, i.e. $44 \mathrm{~dB}$ and also for this set of measurements, with partial window opening, the characteristic amplitudes of tonal frequencies were shifted into higher frequencies. At higher car speeds, two specific tonal frequencies of mechanical nature were identified. With open windows (or window), these tonal frequencies are masked by the source of strong aerodynamic low-frequency noise.

Variation of the A-weighted sound pressure level (SPLA), variation of C-weighted sound pressure level (SPLC), variation of Z-weighted sound pressure level (SPLZ), and also frequency variation as a function of analysed car speeds is presented in Fig. 5. From Fig. 5, it is obvious that the highest energy values of the tonal low-frequency noise with fully open windows occur at car speeds from $80 \mathrm{~km} / \mathrm{h}$ to $130 \mathrm{~km} / \mathrm{h}$. The measured levels are close to the threshold of pain. Nonnegligible energy present values at lower as well as higher cars speeds. A significant difference in energy values is observed when an acoustic weighting is used, i.e. an artificial correction of human being exposition with the exception of different sound perception at the defined frequency bandwidth. From that, the question can be raised, is it more accurate to use C- or Z- weighting in the evaluation of powerful acoustic vibration in very low-frequency bands?

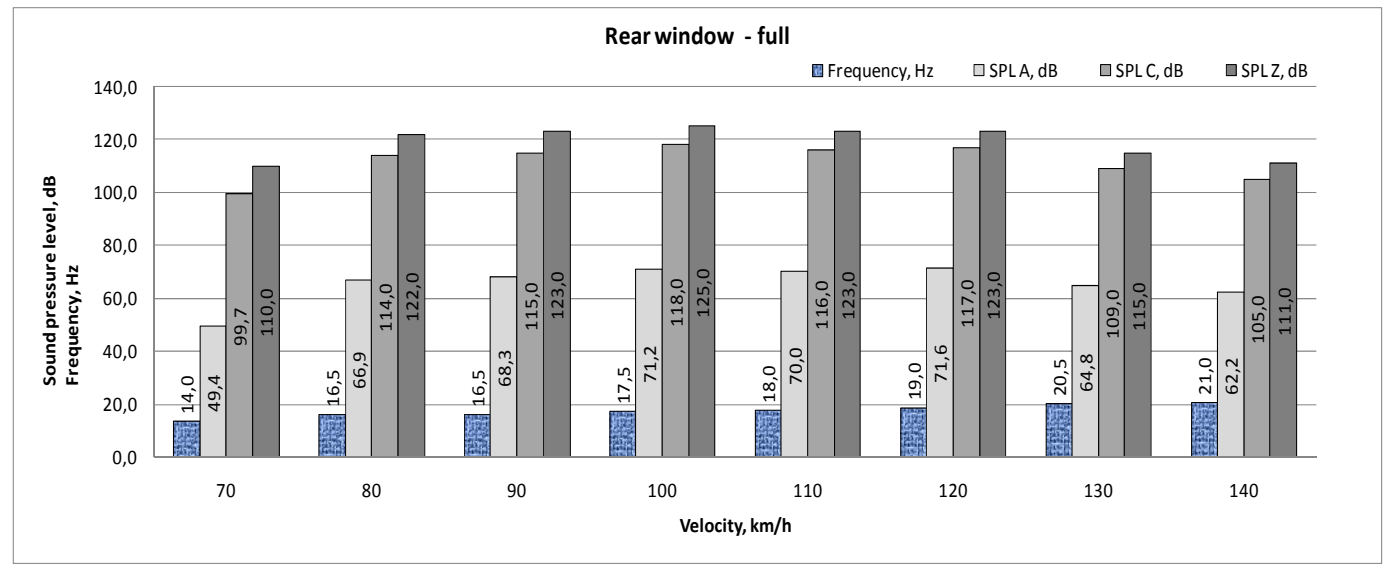

Figure 5: The levels of A-, C- and Z- weighted sound pressure and variation of the frequency as a function of the car speed

In Fig.5 it can be seen that a variation of the speed and the corresponding characteristic frequency of the tonal noise is changed from the region of infrasound into the range of audible sound. 


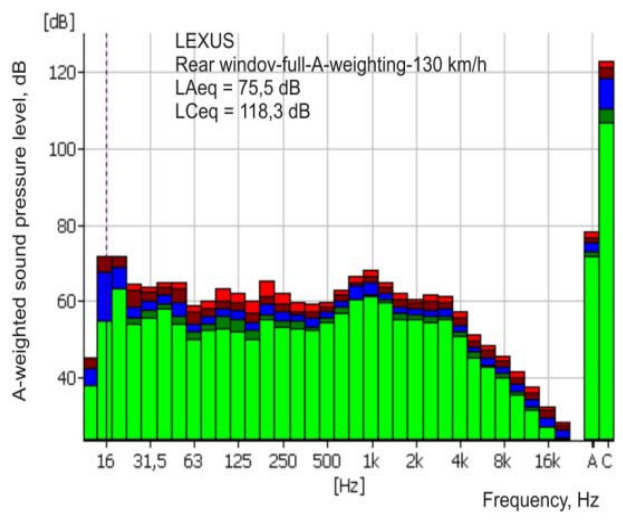

a)

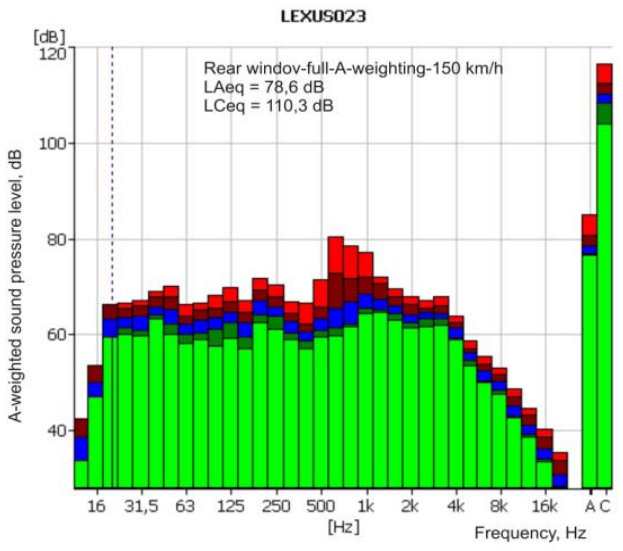

b)

Figure 6: Third-octave analysis of the A- and C-weighted sound pressure level of the same acoustic signal with the car speed: a) $130 \mathrm{~km} / \mathrm{h}$; b) $150 \mathrm{~km} / \mathrm{h}$

In the interior of the LEXUS the measurements were carried out following the same methodology as in the previous analysis, i.e. the third-octave CPB analysis using an A-weighting as well as C- weighting measured at two car speeds, i.e. at $130 \mathrm{~km} / \mathrm{h}$ (Fig. 6a) and $150 \mathrm{~km} / \mathrm{h}$ (Fig. 6b). Even though different cars are used, this analysis shows that in the interior of the car the generation of significant low-frequency acoustical vibration is induced. This phenomenon is similar to the one analysed in the previous section. This significant tonal noise, with a centre frequency of $16 \mathrm{~Hz}$, can be identified from Fig.6a, i.e. in third-octave band CPB analysis. Analogically to the previous measurements (inside of the NISSAN), the tonal frequency varied with car speed. Similarly, the sound pressure level measured in the LEXUS corresponded to the one measured in the NISSAN. Again it needs to be emphasized that the perception of strong lowfrequency noise was much more significant than the A- weighted level. The perception corresponded more to the C- weighted level, probably also because of the fact that C-weighting is close to the threshold of pain. Furthermore, the analysed low-frequency, energy rich, acoustical vibration is close to the threshold of pain. Increasing the speed above $130 \mathrm{~km} / \mathrm{h}$, the specific tonal low-frequency acoustic vibration generated by air entering the interior of the car is decreased and the noise induced from tyre and aerodynamic noise became dominant (Fig. 6b).

\section{NOISE WITH STRONG LOW-FREQUENCY CONTENT AND DISCUSSION}

The noise acting on human beings has s physical effects on human hearing as well as a physiological effect on the whole human organism, which mainly depend on the power of the sound energy, and psychological effects which depend on the qualitative parameters of the noise (sound), such as spectral composition (sharpness), roughness, signal information, time distribution, tonal content, speech-to-noise ratio, etc $[6,10,11]$.

Research has shown that the frequency A-weighting alone is not sufficient to assess sounds characterized by tonality, impulsiveness or strong low-frequency content. To estimate the long-term annoyance response of a community to sounds with some of these special characteristics, an adjustment in decibels is added to the A-weighted sound exposure level or Aweighted equivalent continuous sound pressure level. Also, research has shown that different transportation sounds or industrial sounds evoke different community annoyance responses for the same A-weighted equivalent continuous sound pressure level [15]. 
Investigations have shown that the perception and effects of sounds differ considerably at low frequencies as compared to mid or high frequencies. The main reasons for these differences are $[3,11,13,15]$ a weakening of pitch sensation as the frequency of the sound decreases below $60 \mathrm{~Hz}$, while sound energy acting on the ear and the whole body is not changeable; perception of sounds as pulsations and fluctuations, where the amplitude can be changed in the range of approximately $6 \mathrm{~dB}$; a much more rapid increase in loudness and annoyance with increasing sound pressure levels at low frequencies than at mid or high frequencies; complaints about feelings of ear pressure; low frequency noise can mask higher frequencies more than high frequencies are able to mask the low frequencies; low frequency noise can cross large distances without significant energy loss from atmospheric and ground attenuation; low frequency noise can cause subjective reactions in humans; ear defenders are less effective at low frequencies; annoyance caused by secondary effects like the rattling of buildings elements, windows, and doors and less sound transmission loss at low frequencies than at mid or high frequencies [1, 2].

For the assessment of sounds with strong very low-frequency content, the rating procedures should be modified. The measurement location may be changed and the frequency weighting is affected since sounds with strong, very low-frequency content induce a greater annoyance than is predicted by the A- weighted sound pressure level [2, 6, 13, 15]. The strong low-frequency content of acoustic vibration very often contains tonal components, and therefore it is more suitable to use CPB analysis or better FFT analysis in the frequency range from $10 \mathrm{~Hz}$ to $100 \mathrm{~Hz}$. For the assessment of acoustic vibration with strong low-frequency content in the frequency range from $10 \mathrm{~Hz}$ to $100 \mathrm{~Hz}$, the C- or Z- weighting should be used rather than the Aweighting and in the assessment criteria of the low-frequency noise, considerations on the basis of sound measurement inside of the protected space rather than outside environment, namely the generation of standing waves in the protected space.

\section{CONCLUSION}

The experiments, and even the personal participation in the experiments, show that the energy of strong acoustic vibration of low-frequency content cannot be correctly evaluated using A-weighting. The main reason is that this filter attenuates the energy severity of the acoustic vibration acting on the human being. The strong energy exposition requires the application of $\mathrm{C}$ - or Z- weighting, in which the sound pressure levels are in closer agreement with the threshold of pain. The results and analysis show that the executed experiments are closer to the evaluation methodology used in other developed countries. It can be concluded that weaker low-frequency acoustic waves can also generate standing waves and so amplify the energy exposition of human beings. The presented recommendations for the evaluation of low-frequency acoustic waves in protected spaces should be taken as a contribution to current knowledge about noise evaluation, as well as a stimulus for the technical community, since the correct evaluation of this type of noise can help to reduce the danger of poor health and reduction of comfort in human beings.

\section{ACKNOWLEDGEMENTS}

The article is published with the support of the Scientific Grant Agency of the Ministry of Education of Slovak Republic and the Slovak Academy of Sciences (VEGA) 1/0135/12.

\section{REFERENCES}

[1] G. P. Berg, "Low Frequency Noise and Vibration and its Control", 11th International Meeting Maastricht, The Netherlands (2004).

[2] British Wind Energy Association, “Low Frequency Noise and Wind Turbines”, Technical Annex (2005). 
[3] N. Broner and H. G. Leventhall, “Annoyance loudness and unacceptability of higher level low frequency noise”, J. Low Frequency Noise Vibr., 4(1), pp. 1-11, (1985).

[4] R. Darula and S. Žiaran, "Influence of Specific Noise on Driver's Comfort", Proceedings of the $5^{\text {th }}$ International Symposium Material - Acoustics - Place 2010, pp. 55-59, Zvolen (2010).

[5] B. Davidsen, "Low frequency Noise Emission from Wind Farms", Potential Health Effects Report number: 09.2819 rev.3, (2009).

[6] M. Mirowska, "Results of measurements and limits proposal for low frequency noise in the living environment”, J. Low Frequency Noise Vib., 14, pp. 135-141 (1995).

[7] D. Piorr and K. H. Wietlake, "Assessment of low frequency noise in the vicinity of industrial noise sources”, J. Low Frequency Noise Vib., 9, 116 (1990).

[8] M. L. S. Vercammen, “Low-frequency noise limits”, J. Low Frequency Noise Vib., 11, pp. 7-12 (1992).

[9] S. Ziaran, “Acoustics and Acoustic Measurements", in Physical Methods, Instruments and Measurements, [Ed. Yuri M. Tsipenyuk], in Encyclopedia of Life Support Systems (EOLSS), Developed under the Auspices of UNESCO, EOLSS Publishers, Oxford, UK, [http://www.eolss.net] (2005).

[10] S. Ziaran, "Protection of human being against vibration and noise" - in Slovak, Monograph, Issued by Slovak University of Technology Bratislava, (2008).

[11] S. Ziaran, “Analysis of annoying low frequency noise boiler-rooms”, Proceeding from International Congress InterNoise 09, Ottawa (2009).

[12] S. Ziaran, "Effects of low-frequency noise in closed space on the human", In InterNoise 2011: 40th International congress and exposition on noise control engineering. Osaka/Japan (2011).

[13] S. Ziaran, S.: Low frequency noise from the open window in a moving car and its effect on human being. InterNoise 2012: 41th International congress and exposition on noise control engineering. New York/USA (2012).

[14] S. Ziaran and R. Darula and J. Oreský, "Noise induced by window opening in a moving car" - in Slovak. In: Noise and vibration in practice. Proceedings of the 17th international acoustic conference, Bratislava (2012)

[15] ISO 1996-1 Acoustics. Description, measurement and assessment of environmental noise. Part 1: Basic quantities and assessment procedures. ISO Geneva 20 (2003). 